\title{
Single Stage Multiple Stenting in Takayasu's Arteritis -Case Report-
}

\author{
Shinya Miyamoto, ${ }^{1}$ Henry Wu, ${ }^{2}$ Takeshi Kubo, ${ }^{3}$ Kenji KaWAguchi, ${ }^{3}$ \\ Takafumi IDE, ${ }^{1}$ Nobuhiko TAKEMURA, ${ }^{1}$ and Shigeru NEMOTO ${ }^{3}$ \\ ${ }^{1}$ Department of Neurosurgery, Tokyo Metropolitan Bokutoh Hospital, Tokyo; \\ ${ }^{2}$ Keck School of Medicine, University of Southern California, Los Angeles, California, USA; \\ ${ }^{3}$ Department of Endovascular Neurosurgery, Toranomon Hospital, Tokyo
}

\begin{abstract}
A 32-year-old Filipino female presented with Takayasu's arteritis manifesting as an abrupt onset of syncope. Physical examination revealed diminished consciousness, right hemiparesis, and a large discrepancy in blood pressure between the upper and lower extremities. Magnetic resonance imaging revealed cerebral infarcts in the left basal ganglia and the left temporal lobe. Angiography revealed complete occlusion of the left common carotid artery and severe stenosis of the brachiocephalic artery, the right common carotid artery, and the left subclavian artery. Based on the clinical examination and studies, the diagnosis was Takayasu's arteritis, type I. The patient's condition stabilized after 2 months of prednisone and anti-platelet therapy. Single stage multiple stenting in the brachiocephalic artery, the right common carotid artery, and the left subclavian artery was then performed using high pressure inflation to dilate the arteries due to the remarkably rigid lesions that resulted from extensive and diffuse fibrosis throughout the vessel walls. Although a small intimal flap occurred during inflation of the left subclavian artery, re-dilation was possible with the stent. Even with evidence of notable recovery in blood pressure and cerebral blood flow, no further neurological improvement was observed. In view of the favorable short- and intermediate-term results, single stage multiple stenting may be the optimum treatment option for first-line stent-supported angioplasty in patients with Takayasu's arteritis.
\end{abstract}

Key words: Takayasu's arteritis, single stage multiple stenting, stent-supported angioplasty, re-stenosis, intravascular ultrasound

\section{Introduction}

Takayasu's arteritis is a non-specific, idiopathic, chronic inflammatory arteritis involving the aorta and its major branches, ${ }^{4)}$ and is prevalent among young, Asian females. ${ }^{6)}$ The inflammation observed in Takayasu's arteritis involves the tunica media and the tunica adventitia of blood vessel walls and also affects the vasa vasorum, producing a diffuse or nodular fibrosis. ${ }^{5)}$ Takayasu's arteritis often induces cerebrovascular accidents as well as ischemic heart disease, cardiac failure, aneurysm, renal failure, retinopathy, and hypertension. ${ }^{10,15,18)}$ The overall survival percentage in patients with Takayasu's arteritis at 10 years af-

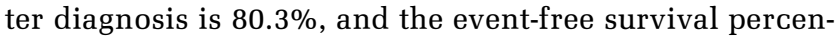
tage at 10 years is $61.6 \% .{ }^{15)}$ However, the treatment for Takayasu's arteritis has not been well established to date. Endovascular interventions, such as percutaneous transluminal balloon angioplasty (PTBA), have shown success in relieving stenotic lesions of major arteries in recent stu- dies. ${ }^{7)}$ Another alternative in the treatment of Takayasu's arteritis is stent-supported angioplasty, but evidence for the efficacy of single stage multiple stenting is limited. Here, we describe a successful treatment using single stage multiple stenting in the brachiocephalic trunk, the right common carotid artery, and the left subclavian artery after acute cerebral infarctions in a young, female patient with Takayasu's arteritis.

\section{Case Report}

A 32-year-old, right-handed, Filipino female suffered sudden onset of altered mental status and was found collapsed at the entrance of her apartment. She was transported to our emergency room in an obtunded state and did not respond to verbal commands. An internist suspected that the patient had suffered from an epileptic attack or an acute onset of encephalopathy, and she was emergently admitted. The patient developed right hemiparesis within

Received March 28, 2011; Accepted June 27, 2011

Author's present address: S. Miyamoto, DDS, MD, PhD, Department of Pathology, University of Oklahoma Health Sciences Center, Oklahoma City, Oklahoma, USA. 


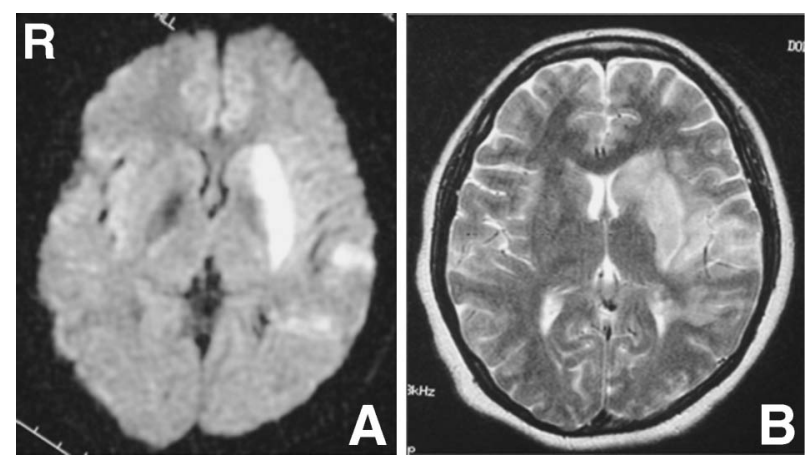

Fig. 1 Diffusion-weighted (A) and $\mathrm{T}_{2}$-weighted (B) magnetic resonance images depicting high intensity signals in the left caudate head, the putamen, and the temporal lobe. Acute left basal ganglia and temporal lobe infarctions were confirmed.

a few hours after admission. Diffusion-weighted and $\mathrm{T}_{2^{-}}$ weighted magnetic resonance imaging revealed left basal ganglia and temporal lobe infarctions (Fig. 1). The patient was referred to the Department of Neurosurgery for further management.

The patient's past medical history included multiple episodes of loss of consciousness at the ages of 16, 18, and 21 years, as well as a history of an ovarian hemorrhage at the age of 31 years. Furthermore, the patient's family history included frequent loss of consciousness in both the patient's mother and sister. Physical examination revealed blood pressure of $67 / 51 \mathrm{mmHg}$ in the upper extremities and $125 /$ not available $\mathrm{mmHg}$ in the lower extremities. The radial pulses were absent bilaterally. Auscultation detected no murmurs or rales over the chest, and no bruits over the abdomen. However, carotid bruits were present bilaterally. Neurological examination showed the patient's level of consciousness was 9 (E3 V2 M4) using the Glasgow Coma Scale. The patient had reactive, isocoric pupils, and evidence of right hemiparesis (motor strength of $1 / 5$ in the upper extremity and 2/5 in the lower extremity). No other significant physical findings were noted.

Laboratory data were remarkable for the presence of elevated markers of inflammation, including erythrocyte sedimentation rate (ESR) of $71 \mathrm{~mm} / \mathrm{hr}$ (normal, 0-9 $\mathrm{mm} / \mathrm{hr}$ ) and C-reactive protein (CRP) level of $1.62 \mathrm{mg} / \mathrm{l}$ (normal, 0-0.36 mg/l). Computed tomography (CT) with contrast medium of the chest and subsequent aortography revealed severe stenosis of the brachiocephalic artery, the right common carotid artery, and the left subclavian artery, in addition to complete occlusion of the left common carotid artery (Fig. 2A, B). Single photon emission computed tomography (SPECT) of the brain showed notably poor blood flow to the left frontal and temporal lobes (Fig. 2C, D). Under a diagnosis of vascular arteritis, intravenous injection of prednisone at $1 \mathrm{mg} / \mathrm{kg}$ daily was started, which was tapered down over one month. Aspirin (330 mg orally administered daily), ticlopidine (100 mg orally administered twice a day), and edaravone (30 mg intravenously administered daily) were also initiated for the cerebral infarctions.

Her level of consciousness gradually improved, but she was confirmed to have severe, pure motor aphasia. With the help of physical and speech therapies during hospitalization, the right hemiparesis and motor aphasia gradually improved to the point where the patient could walk with assistance and to communicate in her native language and in English within a 2-month time span. After the patient had recovered from the acute phases of stroke and vascular arteritis as evidenced by an ESR of $7 \mathrm{~mm} / \mathrm{hr}$ and a CRP level of $0.03 \mathrm{mg} / \mathrm{l}$, stent-supported angioplasty was performed on the 72nd day of her hospital stay in an effort to prevent future cerebrovascular accidents.

Throughout the procedure, the patient was given 5,000 units of heparin to maintain an activated coagulation time between 250 and 300 seconds. Quantitative right brachiocephalic angiography was undertaken to measure the diameter of the vessels and the length of the lesions. Protection balloon systems were placed in the right vertebral artery and the internal carotid artery to prevent distal emboli. Pre-dilation was performed with a $4 \mathrm{~mm} \times 30$ $\mathrm{mm}$ balloon catheter, and an $8 \mathrm{~mm} \times 60 \mathrm{~mm} \mathrm{Smart}{ }^{\circledR}$ Stent (Cordis Corporation, Bridgewater, New Jersey, USA) was deployed in the brachiocephalic trunk and the right common carotid artery. The stent had a small waist at the site of the lesion so that dilation could be performed with a 6 $\mathrm{mm} \times 40 \mathrm{~mm}$ balloon catheter and an $8 \mathrm{~mm} \times 20 \mathrm{~mm}$ balloon to obtain the optimal result. Despite the use of high pressure inflation in two attempts, the brachiocephalic trunk and the right common carotid artery could not be completely dilated. However, angiography showed satisfactory reconstruction of the brachiocephalic trunk from the ostium up to the common carotid artery. Next, a $7 \mathrm{~mm}$ $\times 13.5 \mathrm{~mm}$ Palmaz ${ }^{\circledR}$ Stent (Cordis Corporation) was deployed across the lesion in the left subclavian artery. High pressure balloon inflation was performed within the stent after the placement. An arterial dissection was confirmed in the wall at the distal end of the stent, so another $8 \mathrm{~mm} \times 12.7 \mathrm{~mm}$ Palmaz ${ }^{\circledR}$ Stent was implanted in the dissected portion of the vessel. High pressure balloon inflation was performed within the second Palmaz ${ }^{\circledR}$ Stent. Good distal flow was achieved in both the right common carotid and the left subclavian arteries (Fig. 3A).

Heparin at 1,000 units per hour was continued for the next 24 hours. Oral aspirin (330 mg administered daily) was resumed thereafter. Slight oozing of blood was present at the left femoral site during heparinization, but completely resolved after discontinuation of the heparin infusion. Although no apparent neurological improvements were achieved after stent-supported angioplasty, both the right and left radial arteries had become well palpable, and the blood pressure was elevated to $94 / 56 \mathrm{mmHg}$ in the right upper extremity and $104 / 60 \mathrm{mmHg}$ in the left upper extremity. CT did not detect post-procedural hemorrhage by reperfusion or cerebral infarction due to microemboli. Post-stenting SPECT also confirmed absence of hyperperfusion secondary to the single stage multiple vascular dilations (Fig. 3E, F). The patient continued rehabilitation for approximately one month, and subsequently returned to her native country. 

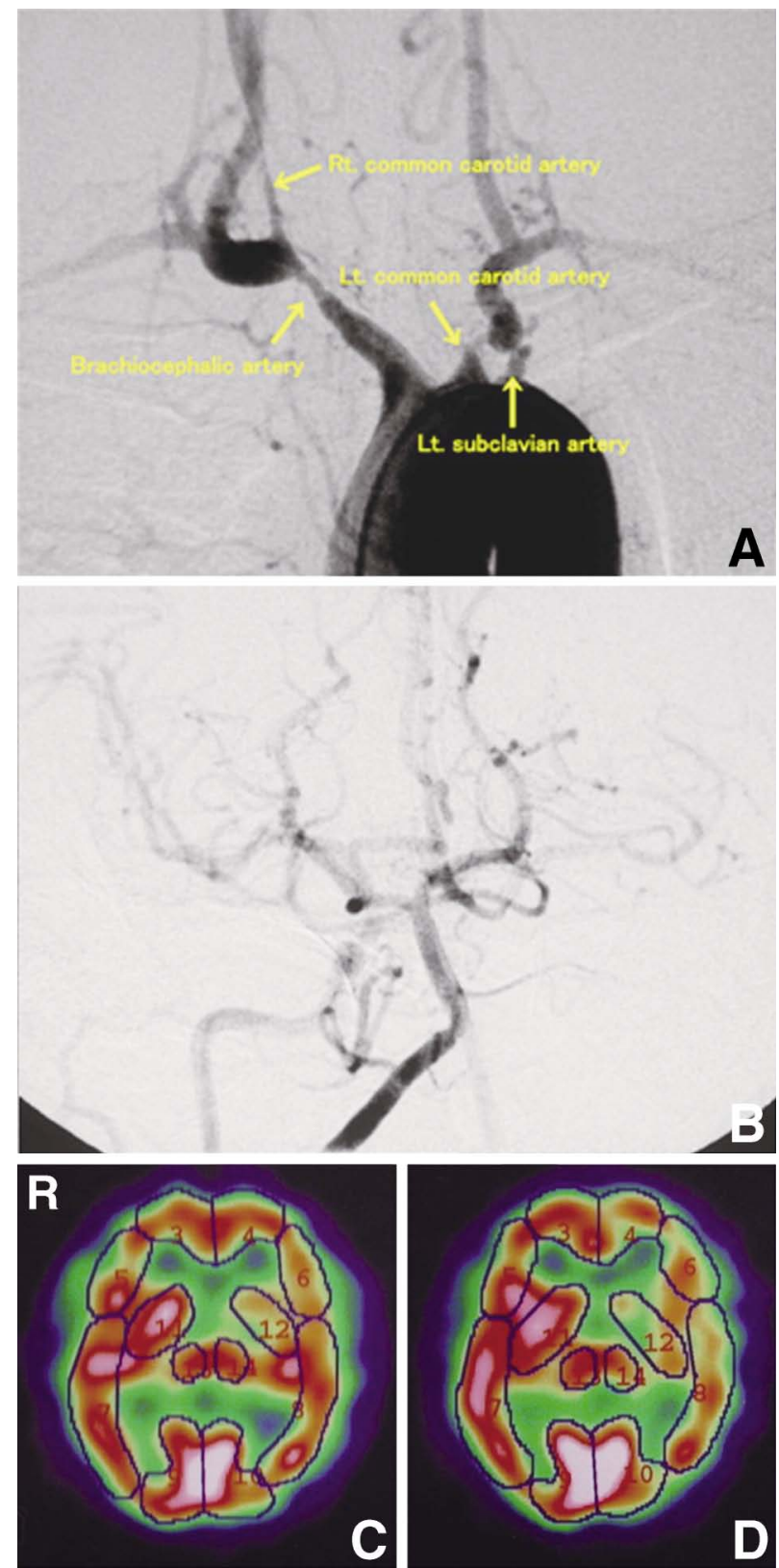

Fig. 2 A: Aortogram revealing severe stenosis of the brachiocephalic artery, the right common carotid artery, and the left subclavian artery, as well as complete occlusion of the left common carotid artery. B: Angiogram showing that the blood flow in the left hemisphere was supplied through the anterior communicating artery from the right internal carotid artery. C, D: Single photon emission computed tomography scans showing a notable decrease in cerebral blood flow to the left basal ganglia and the left temporal lobe, corresponding to the infarcted areas (C), and further decrease in blood flow in the corresponding infarcted areas with acetazolamide loading (D).

\section{Discussion}

The diagnosis of Takayasu's arteritis in asymptomatic patients is challenging to most physicians since only $33 \%$
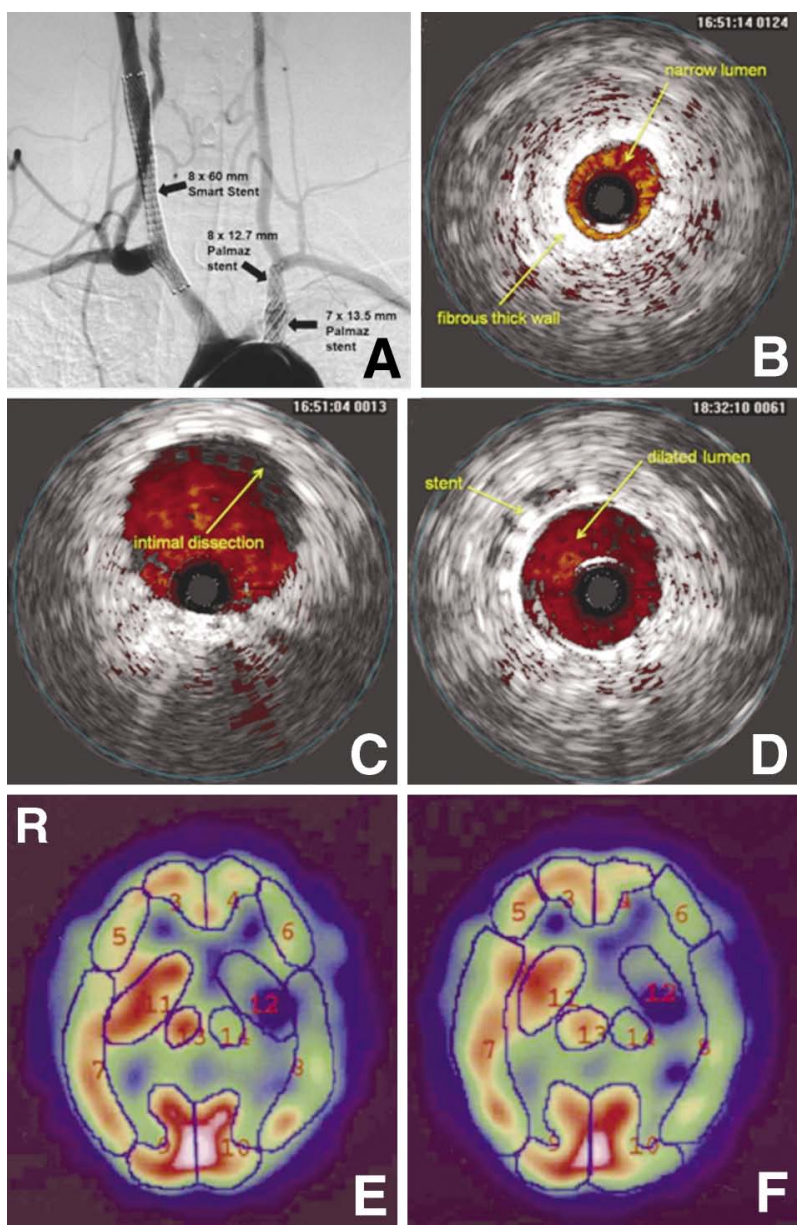

Fig. 3 A: Post-stenting angiogram confirming correct stent placements and improved return of blood flow after treatment. The left common carotid artery was not dilated due to its complete occlusion. B-D: Intravascular ultrasonograms depicting the thick fibrous vessel wall and the narrow lumen of the Takayasu's arteritis lesion (B), the intimal dissection that occurred during high pressure inflation (C), and the dilated lumen and correct deployment of the stent (D). E, F: Post-stenting single photon emission computed tomography scans without (E) and with (F) acetazolamide loading confirming absence of hyperperfusion in the brain.

of patients with Takayasu's arteritis have systemic symptoms on presentation. ${ }^{6}$ ) Our patient met 4 of the 6 criteria for the classification of Takayasu's arteritis, as defined by the 1990 guidelines from the American College of Rheumatology. Since the aorta was not involved, the patient was classified as the most common type, type I, occurring in $50 \%$ of patients with Takayasu's arteritis, ${ }^{13)}$ based on the classification guidelines from the International Conference on Takayasu's Arteritis in 1994.

Some patients with Takayasu's arteritis $(20 \%)$ present with cerebrovascular symptoms, such as disequilibrium, syncope, visual disturbances, transient ischemic attack, seizures, and altered sensorium. ${ }^{10,14,15,18)}$ Severe hypertension, severe functional disability, and evidence of cardiac involvement are good predictors of either death or a major 
Table 1 Comparison between treatment methods for Takayasu's arteritis

\begin{tabular}{lccc}
\hline & Bypass surgery & PTBA & Stenting \\
\hline Technical difficulty & high & low & fair \\
Risk of procedure & high & low & fair \\
Frequency of restenosis & low & high & fair \\
Invasiveness & high & low & low \\
Average cost & high & low & high \\
\hline
\end{tabular}

PTBA: percutaneous transluminal balloon angioplasty.

event during follow up of the patient. ${ }^{15}$ One study found that $6.8 \%$ of patients with Takayasu's arteritis reported complications of hemiplegia, and $4.5 \%$ of patients with Takayasu's arteritis reported loss of vision at the last follow-up visit. ${ }^{15)}$ Patients with no complications or with a single, mild complication at diagnosis had a higher eventfree survival rate compared to those with severe, single complication, or multiple complications at 5 years $(97.0 \pm$ $2.9 \%$ and $59.7 \pm 7.3 \%$, respectively). ${ }^{15)}$ Cerebrovascular events contribute to $20 \%$ of the deaths in patients with Takayasu's arteritis. ${ }^{1)}$ Therefore, managing the extent of arteritis and the prevention of major, cerebrovascular events are important considerations for patients with Takayasu's arteritis.

To manage active arteritis, an initial dose of corticosteroid administered at $1 \mathrm{mg} / \mathrm{kg} / \mathrm{day}$ for one month, and then tapered down to less than $10 \mathrm{mg} / \mathrm{day}$, is the initial mainstay of medical therapy for patients with Takayasu's arteritis. ${ }^{4,9)}$ However, studies report that only $20 \%$ of patients with Takayasu's arteritis maintain remission for 6 months on less than $10 \mathrm{mg}$ prednisone. ${ }^{4)}$ Since no medical therapies can reverse established vascular stenosis, ${ }^{4}$ the combination of medical therapy and subsequent revascularization is crucial in patients with Takayasu's arteritis.

Stent-supported angioplasty has recently emerged as the treatment of choice for stenotic lesions in patients with Takayasu's arteritis (Table 1). Stents not only assist in achieving larger luminal diameters, but may also eradicate or remarkably decrease gradients across stenotic lesions. ${ }^{2)}$ Re-stenosis has been observed in $44.4 \%$ of lesions treated with only angioplasty, but only $17.6 \%$ of lesions showed re-stenosis with the use of angioplasty with stenting. ${ }^{7)} \mathrm{On}$ the other hand, the use of stents may not yield long-term vessel patency in patients with Takayasu's arteritis as compared to the use of stents in the treatment of atherosclerosis. ${ }^{8)}$ However, all recurrent stenoses in patients with Takayasu's arteritis have been treated successfully by reintervention without significant complications. ${ }^{7)}$

The key to successful stenting in patients with Takayasu's arteritis is to evaluate the lesions frequently during the intervention and to dilate the rigid vessels carefully with adequately high pressure. Compared to atherosclerotic lesions, the lesions in patients with Takayasu's arteritis are very rigid due to their extensively diffuse, fibrotic, eccentric, and calcified nature. ${ }^{11,16)}$ Although arterial lesions of Takayasu's arteritis have similar luminal diameter of stenosis compared to arterial lesions of atheros- clerosis before PTBA, they require higher balloon inflation pressure to be dilated, resulting in greater risk for residual stenosis after PTBA. ${ }^{17)}$ In one study, the required mean inflation pressure was $5.5 \mathrm{~atm}$ for arterial lesions of atherosclerosis compared to $9.9 \mathrm{~atm}$ for arterial lesions of Takayasu's arteritis. ${ }^{17)}$ Since higher balloon inflation pressure causes more back pain, more frequent intimal dissection, bradycardia, and hypotension, close monitoring is required during dilation of balloons and stents. In our patient, minimal intimal dissection occurred in the left subclavian artery, and insertion of an additional stent allowed sufficient dilation.

Intravascular ultrasonography (IVUS) was frequently used during angioplasty as a great diagnostic tool to evaluate the wall of blood vessels, to determine the ideal positions of the stents, and to re-evaluate the final stent placement (Fig. 3B, D). The intimal flap that occurred during high pressure inflation and the well-opposed stents that increased the luminal diameter were well depicted by IVUS (Fig. 3C). In addition to selection of an appropriately sized vessel and using anti-platelet therapy to prevent blood coagulations, IVUS is a remarkably helpful tool in deploying a stent safely in patients with Takayasu's arteritis.

Since involved aortic segments in patients with Takayasu's arteritis have smooth and regular intimal surfaces, ${ }^{3)}$ deployment of a balloon catheter or a stent in arterial lesions of Takayasu's arteritis can be unstable. To avoid stent slipping or stent migration, the stent was repeatedly dilated with moderately high pressure. Distal emboli do not occur as frequently in patients with Takayasu's arteritis as in patients with atherosclerosis, which is attributable to the smooth intima. Accordingly, some endovascular surgeons claim that it is unnecessary to protect against distal emboli in patients with Takayasu's arteritis. However, given the risks and benefits, we recommend placing of a protecting device in the vessel distal to the lesion if this can be feasibly achieved.

Whether single stage deployment is the preferred option to multiple stage deployments remains controversial. Some endovascular surgeons claim that single stage multiple deployment may cause drastic dynamic changes in blood flow, which would subsequently increase the risk of cerebrovascular accidents. Cerebral hemorrhage after bilateral aorta to carotid grafts has been the cause of deaths, ${ }^{12,14)}$ attributed to sudden, excessive blood flow through the graft or to severe autonomic dysregulation that may have been related to bilateral carotid sinus dysfunction as a result of Takayasu's arteritis. ${ }^{12,14)}$ However, we should note that excessive anti-coagulation or antiplatelet medication during and after surgery may also be implicated in the occurrence of these cerebral hemorrhages. In our case, single stage multiple deployments had no apparent adverse events during and after the procedure, possibly as our patient had cerebral infarcts limited to the left basal ganglia and the left temporal lobe. Although single stage deployments are less invasive and are more cost-effective when compared to multiple stage deployments, the risk of post-stenting hyperperfusion is higher than multiple stage deployments. Thus, the tech- 
nique of single stage multiple deployments in the carotid arteries should be used selectively only in patients with small infarcts and the post-stenting hyperperfusion is less likely.

Furthermore, it is important for endovascular surgeons to consider the order of dilation in deploying multiple stents in several aortic branches. Dilating a brachiocephalic trunk before a common carotid artery may precipitate distal emboli. It is generally advisable to dilate a distal artery before a proximal artery, if possible. However, some endovascular surgeons claim that it is permissible to dilate only the proximal part of the lesions instead of whole narrow segments; for instance, it would be permissible to dilate only the common carotid artery in the case of intractable and rigid internal carotid artery stenosis.

Since arterial lesions of Takayasu's arteritis have many different features compared to arterial lesions of atherosclerosis, endovascular surgeons should keep such characteristics of Takayasu's arteritis lesions in mind during angioplasty. Stent-supported angioplasty has many advantages compared to PTBA and vascular surgery. However, the rate of long-term patency and the cost effectiveness of stent-supported angioplasty in patients with Takayasu's arteritis remain unclear. At present, in the absence of randomized trials, it is premature to conclude that stent-supported angioplasty is superior to either PTBA or vascular surgery in patients with Takayasu's arteritis. Nevertheless, in view of the favorable short- and intermediate-term results, stent-supported angioplasty is rapidly becoming the preferred treatment at many medical institutions.

\section{Acknowledgment}

We are deeply grateful to George Hanna for his assistance with the manuscript and for his suggestions.

\section{References}

1) Bali HK, Bhargava M, Bhatta YK, Sandhu MS: Single stage bilateral common carotid artery stenting in a patient of Takayasu arteritis. Neurol India 49: 87-90, 2001

2) Bali HK, Jain AK: Takayasu's arteritis: current status of angioplasty and stenting. Asian Cardiovasc Thorac Ann 7: 339-344, 1999

3) Bezerra Lira-Filho E, Campos O, Lazaro Andrade J, Henrique Fischer C, Godoy Nunes C, Cavalcanti Lins A, Hata F, Carvalho AC: Thoracic aorta evaluation in patients with Takayasu's arteritis by transesophageal echocardiography. J Am Soc Echocardiogr 19: 829-834, 2006

4) Borg FA, Dasgupta B: Treatment and outcomes of large vessel arteritis. Best Pract Res Clin Rheumatol 23: 325-337, 2009
5) Hotchi M: Pathological studies on Takayasu arteritis. Heart Vessels Suppl 7: 11-17, 1992

6) Kerr GS, Hallahan CW, Giordano J, Leavitt RY, Fauci AS, Rottem M, Hoffman GS: Takayasu arteritis. Ann Intern Med 120: 919-929, 1994

7) Lee BB, Laredo J, Neville R, Villavicencio JL: Endovascular management of takayasu arteritis: is it a durable option? Vascular 17: 138-146, 2009

8) Liang P, Hoffman GS: Advances in the medical and surgical treatment of Takayasu arteritis. Curr Opin Rheumatol 17: 16-24, 2005

9) Maksimowicz-McKinnon K, Clark TM, Hoffman GS: Limitations of therapy and a guarded prognosis in an American cohort of Takayasu arteritis patients. Arthritis Rheum 56: 1000-1009, 2007

10) Mwipatayi BP, Jeffery PC, Beningfield SJ, Matley PJ, Naidoo NG, Kalla AA, Kahn D: Takayasu arteritis: clinical features and management: report of 272 cases. ANZ J Surg 75: 110-117, 2005

11) Ozdil E, Parikh DK, Krajcer Z, Angelini P: Stent placement in a patient with Takayasu's arteritis. Cathet Cardiovasc Diagn 38: 373-376, 1996

12) Pajari R, Hekali P, Harjola PT: Treatment of Takayasu's arteritis: an analysis of 29 operated patients. Thorac Cardiovasc Surg 34: 176-181, 1986

13) Petrovic-Rackov L, Pejnovic N, Jevtic M, Damjanov N: Longitudinal study of 16 patients with Takayasu's arteritis: clinical features and therapeutic management. Clin Rheumatol 28: 179-185, 2009

14) Stoodley MA, Thompson RC, Mitchell RS, Marks MP, Steinberg GK: Neurosurgical and neuroendovascular management of Takayasu's arteritis. Neurosurgery 46: 841-852, 2000

15) Subramanyan R, Joy J, Balakrishnan KG: Natural history of aortoarteritis (Takayasu's disease). Circulation 80: 429-437, 1989

16) Tyagi S, Kaul UA, Nair M, Sethi KK, Arora R, Khalilullah M: Balloon angioplasty of the aorta in Takayasu's arteritis: initial and long-term results. Am Heart J 124: 876-882, 1992

17) Tyagi S, Verma PK, Gambhir DS, Kaul UA, Saha R, Arora R: Early and long-term results of subclavian angioplasty in aortoarteritis (Takayasu disease): comparison with atherosclerosis. Cardiovasc Intervent Radiol 21: 219-224, 1998

18) Vanoli M, Daina E, Salvarani C, Sabbadini MG, Rossi C, Bacchiani G, Schieppati A, Baldissera E, Bertolini G: Takayasu's arteritis: A study of 104 Italian patients. Arthritis Rheum 53: 100-107, 2005

Address reprint requests to: Shinya Miyamoto, DDS, MD, PhD, Department of Pathology, University of Oklahoma Health Sciences Center, Suite 1405, 975 NE 10th, Oklahoma City, OK 73104, USA.

e-mail: shinya-miyamoto@ouhsc.edu 